\title{
Context-Aware Cyber Threat Intelligence Exchange Platform
}

\author{
Michael Motlhabi, Phumeza Pantsi, Bokang Mangoale, Rofhiwa Netshiya and Samson \\ Chishiri \\ Council for Scientific and Industrial Research, Pretoria, South Africa \\ mmotlhabi@csir.co.za \\ ppantsi@csir.co.za \\ bmangoale@csir.co.za \\ rnetshiya@csir.co.za \\ schishiri@csir.co.za
}

\begin{abstract}
The ubiquity of network and internet-connected devices has increased exponentially in the past decade. The proliferation of end-user devices has created a lucrative environment for cybercriminals to exploit unsuspecting users at a personal and organizational level. Moreover, businesses and governments are heavily reliant on cyberspace to conduct their business. According to Accenture, in 2019 South Africa saw a spike in cyberattacks on all fronts-banks, Internet Service Providers (ISPs), utilities and eCommerce platforms. This shows that threat actors are continuously looking to exploit new and old vulnerabilities at ever-increasing rates. Furthermore, threat actors are sharing tactics, tools, and procedures to expand their attack surface and to improve the effectiveness of their attacks. Security research tends to be an insular process and rarely do individuals or groups share threat data. This is due to lack of trust, organizational policies, or simply the inability to get the information out to the masses. The idea behind this paper is to design a context-aware threat intelligence exchange platform that encourages collaboration and creates a federated environment amongst different industry stakeholders to share Indicators of Compromise. This paper further aims to define the process of transforming raw Indicators of Compromise into cyber threat intelligence. The platform described in this paper, when implemented, would provide the basic building blocks for developing a highly effective cybersecurity intelligence-sharing system that can improve vulnerability detection and remediation by speeding up the time required to identify/resolve incidents.
\end{abstract}

Keywords: Security Event Management; Security Information Management, Threat Intelligence, Cybersecurity, Collaboration, Data exchange, Indicators of Compromise, TAXI/STIXX, Tactics, Techniques and Procedures

\section{Introduction}

There has been significant improvements to technology and the internet over the years, yielding to the enlargement of communication options thereby an increased reliance on Information Technology (IT). In as much as these improvements have changed human livelihood for the better, they have in turn increased opportunities for cybercriminals. South Africa (SA) is currently ranked 34th out of 108 countries, with a score of 0.417 on the global scale for the Cybersecurity Exposure Index (CEI) published in October 2020 (Frisby, 2020). The CEI is based on data collected from the dark web and the deep web's publicly available sources (Cyber Intelligence House, 2020). According to a report by Accenture, SA has seen an increase in cyber-crimes in recent years with notable spikes in 2019 (Accenture, 2020). The report indicates that several sectors in SA were affected by coordinated cyber-attacks from international threat actors who reused some of their attacks. The force of the attacks included stakeholders in banking, e-commerce, telecommunication provides, Non-Governmental Organizations (NGOs) and provincial utility platforms such as water and sanitation. The Experian Security Breach was one of the cyberattacks which occurred in 2020, affecting approximately 24 million and 793000 thousand local businesses (Cimpanu, 2020) (Burger-Smidt, 2020). In 2021, a most recent cyber-attack that occurred in July, South Africa's largest logistics company, Transnet was targeted. The company was hit by a Death Kitty ransomware which disrupted some of its services (CybercrimeSA, 2014-2021) (TechCentral, 2021).

Consequences of cyberattacks do not only include compromise of intellectual property and loss of data but also disruptions to business operations that may lead to financial loss, reputational damage and regulatory fines. According to a financial stability report by the SA Reserve Bank, cyberattacks continually present a danger to the availability of financial services and the functioning of its infrastructures, thereby creating reputational and direct financial risks (South African Reserve Bank, 2021). The increased adoption of Internet of Things (IoT) technology-based processes within the SA government and private sectors has expanded the threat surface as IoT is not secure by default (Allen, 2021). The Operational Technology (OT), industrial control systems (ICS) and supervisory control and data acquisition systems (SCADA) environments are also part of the idea for connectivity and are by design vulnerable to cybersecurity attacks due to the use of legacy IT systems. The most vulnerable industries to cyberattacks in 2021 as reported by CDNetworks, include but are not limited to government 
agencies, energy companies, healthcare institutions and small businesses (CDNETWORKS, 2021). Compromise of government agencies could lead to exposure of confidential data such as Personally Identifiable Information (PII) i.e., medical health, military or financial data. SA is heavily dependent on a single entity for electricity supply, thus the higher the risk of impact if that entity is compromised (Allen, 2021).

An effective manner to address cybersecurity threats involves sharing best practices, adopting codes of practice for threats and the exchange of information throughout the various sectors. The more that is known about a threat, the easier it is to understand, track and counter it (Lauren \& Irwin, 2015). This paper details a Proof of Concept $(\mathrm{PoC})$ for a cyber threat sharing platform that uses best practices and protocols for threat data exchange and definitions in the SA cyber context. Chapter 2 expands on the current state of the art in the Cyber Threat Intelligence (CTI) domain. Chapter 3 discusses the threat exchange methodology for the PoC. Chapter 4 explains the potential framework for a context-aware CTI system. Chapter 5 focuses on standards and legislative compliance in the SA context and Chapter 6 concludes the paper and provides possible future work and improvements.

\section{State of the Art for Threat Intelligence Platforms}

(Kaspersky, 2021) defined threat intelligence as a method of filtering through large amounts of data to identify and analyze cyber threats that can potentially cause harm. A threat intelligence platform aims to provide organized, analyzed, and refined information regarding potential and current risks that threaten various ICT entities within defined cyberspace (Cascavilla, et al., 2021). The intelligence from these platforms can be embedded into organisational workflows and assist decision-makers in making tactical decisions. Some examples of threat intelligence platforms include:

- The Malware Information Sharing Platform (MISP) - Open-source threat intelligence platform for gathering, sharing, storing, and correlating Indicators of Compromise (loC) of targeted attacks, threat intelligence, financial fraud information, vulnerability information or even counter-terrorism information (MISP Project, 2021).

- The Collective Intelligence Framework (CIF) - Actionable threat intelligence platform focused on speed, performance, and integration (GADGETS, 2021).

- ThreatQ - Threat intelligence platform aimed at providing defenders with the context, customization and collaboration needed for increased security effectiveness and efficient threat operations and management (ThreatQuotient, 2021)

- Anomali ThreatStream - Threat Intelligence Management that automates the collection and processing of raw data, filters out the noise and transforms it into relevant, actionable threat intelligence for security teams (Anomali, 2021)

Cyber threat intelligence sharing provides a proactive manner of creating situational awareness through information sharing, regarding new threats and vulnerabilities. However, this exchange of information heavily relies on trust between stakeholders. A literature survey conducted by (Wagner, et al., 2019), discusses various factors that may hinder or support the process of threat intelligence sharing between stakeholders. These include automation, legal factors, policies, cultural differences, as well as the human role in threat intelligence sharing.

A report published by (ENISA, 2018), presented some of the limitations related to the state and usage of threat intelligence platforms. The authors stated that the amount of data shared tends to be large because of combining data from open-sources, commercial sources as well as private communities. In addition to a large amount of data, there is limited technology enablement to allow end-users to efficiently facilitate the relevancy determination process. Moreover, these platforms have limited analysis and management capabilities to allow users to interpret, analyse, enrich, and react to the threat information received promptly.

The threat intelligence platform analysis done by (Alessandra, et al., 2020), revealed that most of the threat intelligence platforms focus on various specific features such as speed, performance, and visualization of information. These features are dependent on the goal of the platform and as diverse as they are, they are all considered to be threat intelligence platforms mainly because there is no standardized definition for the concept of cyber threat intelligence. 


\section{Cyber threat information exchange}

This section describes the architecture of the repository used to exchange Cyber-Threat Intelligence (CTI). CTI is a collection of intelligence combining Open-Source Intelligence (OSINT), Social Media Intelligence (SOCMINT), Measurement and Signature Intelligence (MASINT), Human Intelligence (HUMINT), and Technical Intelligence (TECHINT) from deep and dark Web (Sari, 2018). Various literature works have demonstrated that CTI can potentially reduce cybersecurity risks and strengthen cyber resilience in both simple and complex cyberspaces (Mtsweni, et al., 2016). Therefore, for any CTI platform to be adequately effective, information sharing about cyber threats must be conducted within a federated community and following clear standards to facilitate automation.

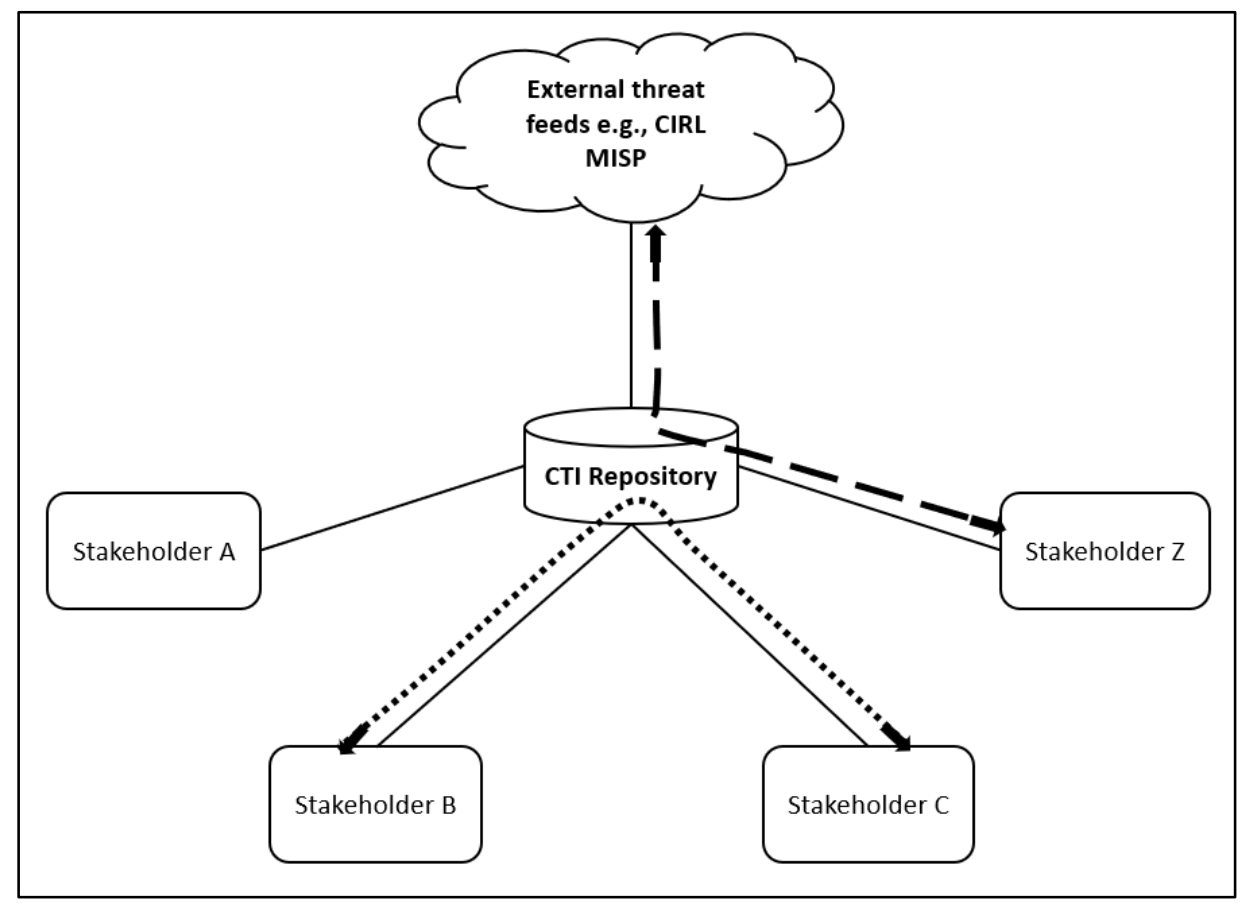

Figure 1: Distributed hub and spoke architecture implementation

Figure 1 shows an architecture that depicts the potential information exchange process of security-related data. Additionally, Figure 1 indicates that a CTI exchange platform should implement a hub and spoke architecture. Hub and spoke is a sharing model in which there is a main data hub (CTI Repo as per Figure 1) where information is coordinated and shared with CTI Stakeholders as seen in Figure 1. The hub and spoke architecture is chosen for the web application because it is advantageous to have a central place for gathering data, and it allows for scaling (Rudman \& Irwin, 2016). The exchange of security incidents in this methodology is bi-directional, with entities alternating between producer and consumer CTI stakeholders. The entity publishing incidents to the central CTI Repo at a particular instance, is the producer of CTI and the one subscribing/receiving from the CTI Repo, is the consumer CTI.

In this model, CTI will be exchanged using the Trusted Automatic Intelligent Information Exchange (TAXII) protocol (OASIS Cyber Threat Intelligence (CTI) TC, 2021). The TAXII protocol will deliver cyber threat intelligence in a simple and scalable manner. Since the CTI exchange platform uses TAXII, stakeholders can choose what information they share and with whom they share CTI with, thus increasing the flexibility of the platform. TAXII supports different sharing models like the hub and spoke, which should be implemented in the CTI exchange platform. TAXII defines the "how", in this implementation, the Hypertext Transfer Protocol Secure (HTTPS) protocol is utilised for transportation because of its security properties.

There are currently no standard formats for loCs defined, however, over the past decade, security experts have developed their formats, such as Incident Object Description Exchange Format (IODEF), OpenIOC3, Cyber Observable Expression (CybOX) and Structured Threat Information eXpression (STIX) (Rudman \& Irwin, 2016). STIX is used to describe information about cyber threats. The goal of STIX is to have a language or representation that allows information to be easily stored, analysed and shared in a consistent manner amongst stakeholders. 
STIX not only allows for the creation of indicators but also other contextual information such as observables, incidents, exploit targets, courses of action, campaigns and threat actors (The Mitre Corporation, 2016). loCs are data that is found in system log entries or files that identify potentially malicious activity in cyberspace (Lord, 2020). The following loCs will be used to detect threats:

1. IP addresses - geographic irregularities, such as traffic from countries or locations where the organization does not have a presence.

2. High authentication failures - attackers use automation to authenticate using phished credentials. A high rate of failed authentication attempts could indicate that an attacker is using multiple incorrect credentials to find credentials that will provide access to a network.

3. Flooded traffic to a specific site or IP - a compromise on a network could make devices create or form part of a botnet, to deploy Distributed Denial of Service (DDoS) attacks.

4. Unusual activity from administrator or privileged accounts, including requests for additional permissions.

5. Domain Name System (DNS) requests anomalies - DNS requests and traffic to command-and-control servers often follows a standard pattern which can serve as a good indicator for suspicious activity.

6. Common Vulnerability and Exposure (CVE) number - CVE number provides a standardized identifier for a given vulnerability or exposure. These can be used to identify vulnerabilities that could be exploited.

The STIX and TAXII protocols provide the CTI exchange platform to leverage the definitions of what are relevant loCs and how they can be shared amongst different stakeholders in the federation. The information being shared by the CTI exchange platform will be in the STIX format and displayed in Hypertext Transfer Markup Language (HTML).

In a web application's Graphical User Interface (GUI), users will gain access to the CTI exchange platform, where they can classify which stakeholder group they are federated to during the sign-up process. Once users have a profile, they can follow relevant organizations and get notified if those organizations upload new threat information. The user profile will determine what CTI will be reported to the user, based on three classes labelled below:

- $\quad$ Strategic - for visualizing broader trends for non-technical audiences.

- Tactical - to outline tactics, techniques and procedures of threat actors and must include technical context about remediation protocols.

- Operational - includes technical details about specific attacks and information about what attack vectors are being used.

To facilitate information exchange between stakeholders in different organizations, MISP will be used ( $\mathrm{NCl}$ Agency, 2017). The MISP platform is used for sharing loCs of targeted attacks for only NATO member nations, governmental and cyber defence organisations. MISP allows for the uploading of information (via text or template), to be manually completed or the PyMISP/ZeroMQ can be used for automation. MISP allows for data export by generating Intrusion Detection System (IDS) rules like Snort and loC formats such as STIX. The platform discussed in this paper is different because it focuses on two key aspects labelled below:

- Southern African Development Community (SADC) - CTI will be shared with stakeholders in the SADC region. This is to increase the cyber security posture of this region. (Accenture, 2020) reports that there has been a visible increase in cyber-attacks in the Southern African region.

- Context-aware network-level information is collected and compared with stakeholders to provide the most relevant and accurate threat response and reporting.

\section{Context-Aware cyber-threat intelligence}

A context-aware cyber-threat intelligence system has visibility to the network elements that are present in the internal environment. Its objectives are to facilitate information sharing, enable automation, and facilitate the generation, refinement and vetting of data through burden-sharing collaboration. A context-aware threat intelligence platform can support timely decision-making, and automate responses to the greatest extent possible, to limit the impact of a potential threat. With this methodology, security teams can get the information they need quickly and from a single source in a rich and contextualized format. 


\subsection{High-level system overview}

Network environments usually generate large amounts of security logs from different network devices and applications. These logs can be collected from internal devices, and cyber-related information can be collectively mapped into cyber intelligence. The platform proposed in this paper, will also consume data from external cyber threat feeds such as MISP, Malware, CVE, malware sites etc. The system will then ingest both the internal and external cyber information to provide a security posture, that is contextualized to a specific organization. The organizations' security posture is shared with key stakeholders who might be impacted. During the CTI sharing process, if any logs are shared between stakeholders, the shared logs will be anonymized.

Since the platform collects network data, the design must allow a stakeholder organization to store cybersecurity data that can either be kept private or shared with others. When data is identified as being private, the system should ensure that the data is never made publicly available to comply with privacy laws and regulations.

\subsection{Navigation and flow}

Figure 2 shows several use cases that the CTI exchange platform can execute. The web application first prompts the user to sign up, and log in, navigate to the dashboard page where the user can perform six major actions. The six capabilities are, Notification and Alarming, Reporting, Collections, Risk Management, Search and Topology. The Administration capability is only accessible from the central CTI Repo (see Figure 1) local instance and is primarily used to configure the platform, check the system health status, and manage licensing.

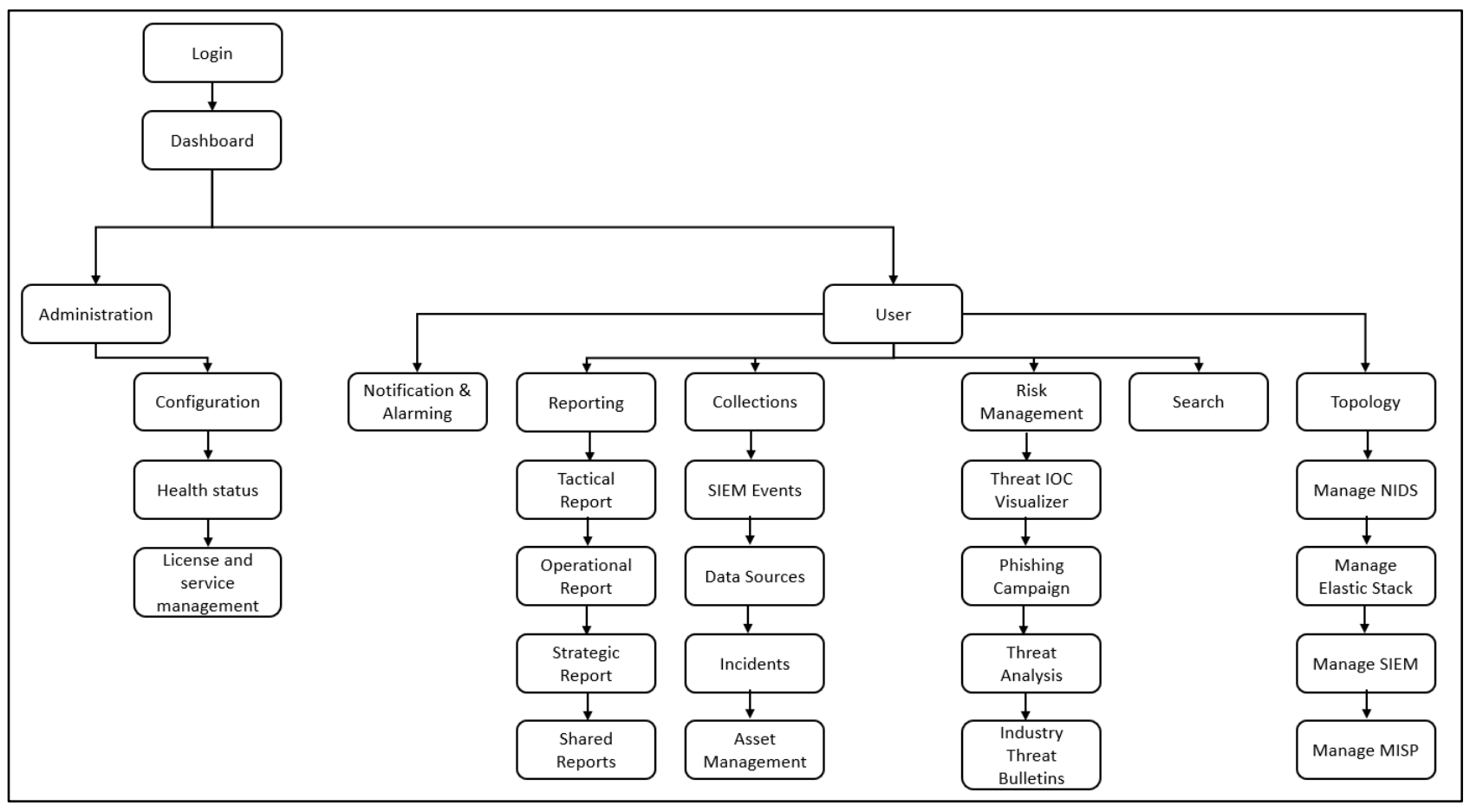

Figure 2: CTI web application capability flow diagram

\subsection{Functional architecture}

The main function of the threat intelligence system discussed here is to create an eco-system that is capable of efficiently capturing loCs and other indicators. A central database will allow for the storage of technical and nontechnical information about malware samples, incidents, attackers, and intelligence. The development of a CTI exchange platform is divided into three categories described in Figure 3. 


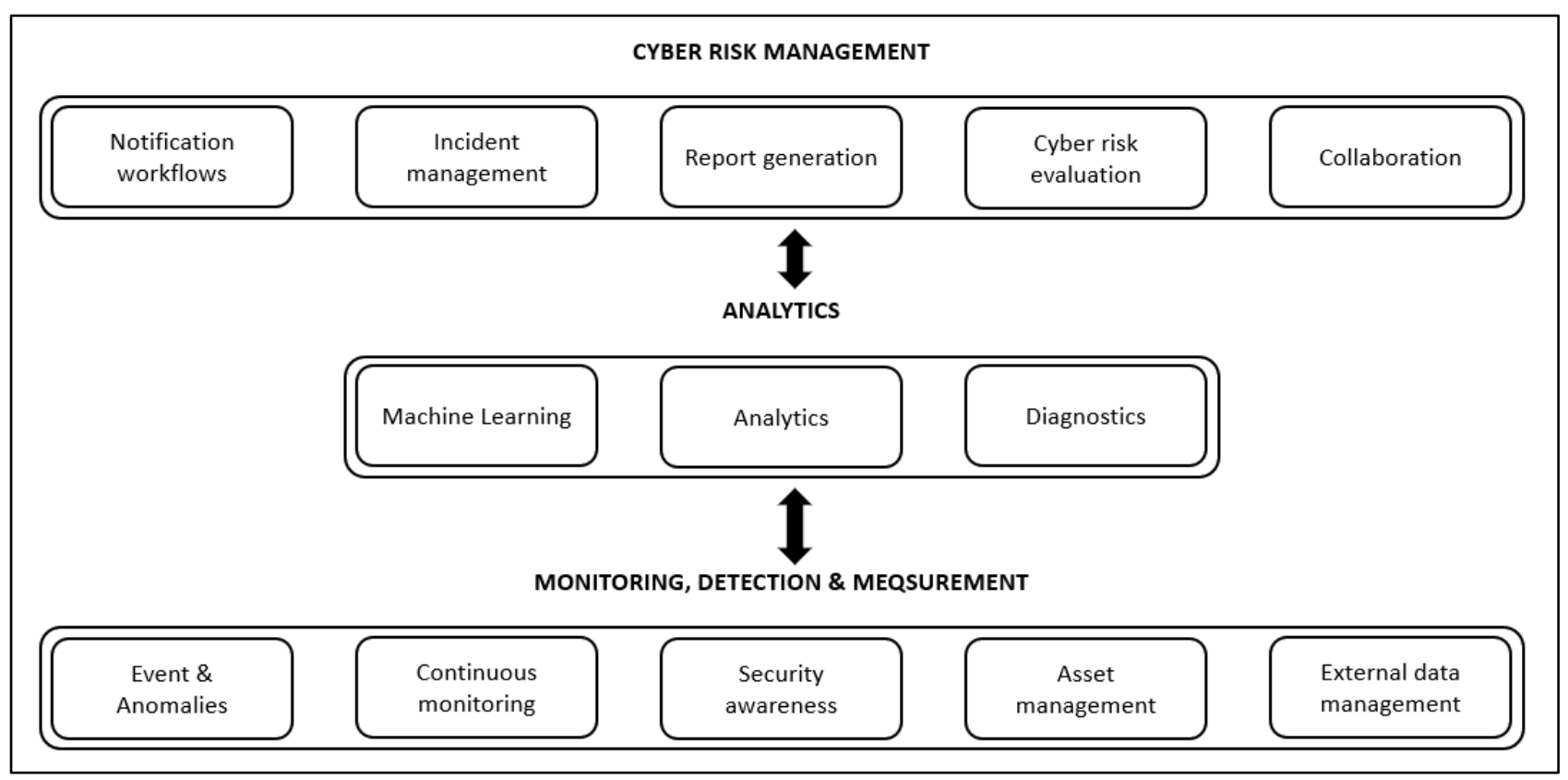

Figure 3: Functional architecture for a context-aware CTI exchange platform

Monitoring, Detection and Measurement: In this segment of the platform, local network variables are collected that are unique to this environment. This enables the CTI sharing platform to accurately monitor attributes that are relevant to the local environment for analysis. Attributes such as network elements events and anomalies form part of the critical system dependencies, that are used to evaluate the security posture in the analysis phase.

Cyber Risk Management: under this category, five distinct attributes can inform the system user about the cybersecurity state, these are functionally explained as follows:

- Cyber Risk Evaluation - a score is generated (in line with CVE scores that measures the likelihood of vulnerabilities being exploited.

- Notification workflows - the detection of a high severity loC will action a notification that will be shared with the stakeholders detailing the specifics of the threats, actors, tactics and/or techniques (Dandurand \& Serrano, 2013).

- Report Generation - a report detailing the threat and the means to remediate that threat will be compiled into three types of reporting for strategic, tactical, and operational audiences.

- Collaboration - different stakeholders will be able to share all the threat intelligence information automatically through the client application installed in the network. The exchanges are necessary to achieve common goals in federated environments and to exploit collaboration opportunities. This objective of quick collaboration and action to remediation is not a technical issue and thus is beyond the scope of these works. It is assumed in this paper that all the stakeholders will comply with the protocols to respond and share their environment specific threat intelligence as quickly as they can respond to them.

Analysis: as data is collected from both the internal environment and the external malware platforms such as the MISP. Machine Learning is used for data analysis and transformation into threat intelligence to find network anomalies (Motlhabi, et al., 2021). loCs are then extracted to show what pertinent threats exist that could compromise the security of the network and other external stakeholders. This section also performs diagnosis, where remediation protocols are defined, reports are populated, and potentially affected stakeholders are notified of the cyber threats.

\subsection{System SIEM process}

A context-aware threat intelligence system should have the capability to analyse local network data. This local analysis ability provides a mechanism for integrating external source threat feed intelligence to provide a security posture that is relevant to the organisation it is deployed in. As such a Security Event Management (SEM) can perform threat monitoring, event correlation, and incident response by analysing the log and event 
data in real-time. Security Information Management (SIM) performs collection, analysis, and reporting on log data (Software Testing Help, 2021). Figure 4 shows a logical set of steps that the platform will follow during its Security, Information and Event Management (SIEM) phase.

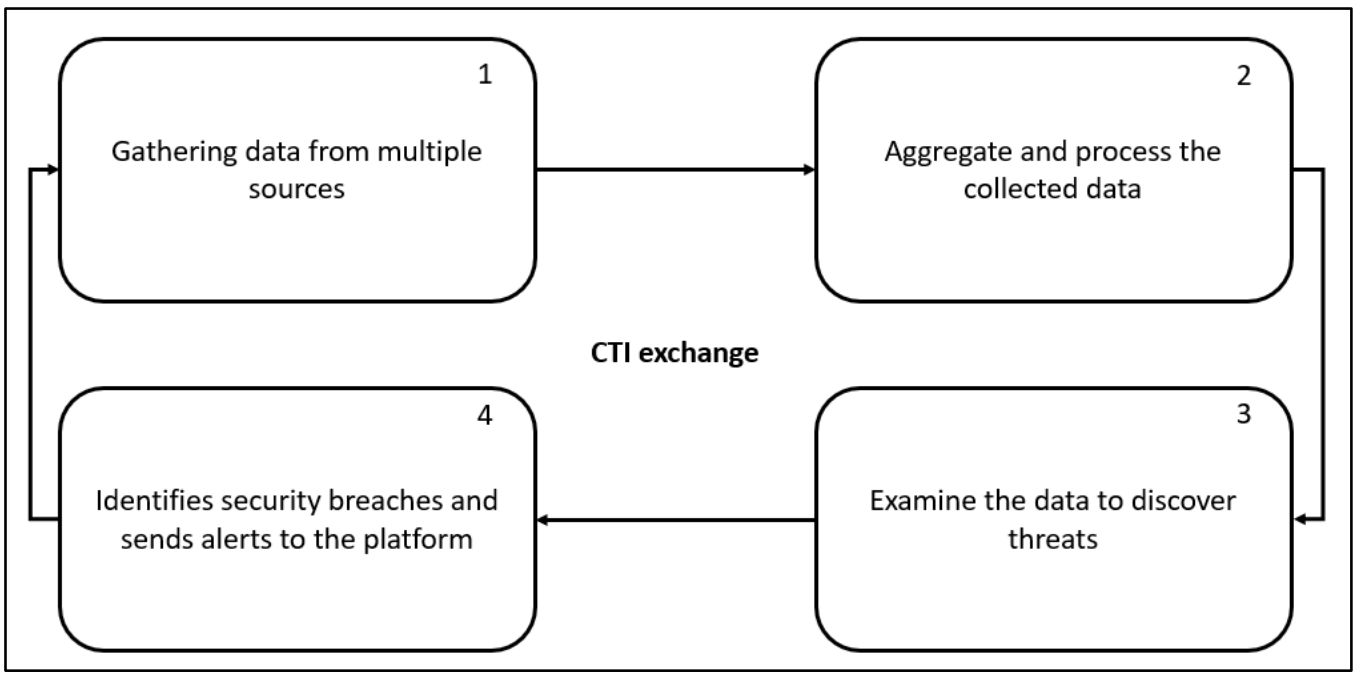

Figure 4: Need to redraw this picture to include external sources

Step 1 cyber threat intelligence data such as malware information, phishing data, loC and other indicators are collected from two sources i.e., the organisations' internal network and the external cyber threat sources that have been collected by the system and/or shared by other organisations. Storage of cybersecurity indicators in a structured formal way is done in this step.

Step 2 raw and rich data is brought together from different sources as public Open-Source Intelligence (OSNIT) feeds and form part of the default configuration of the SIEM function. The threat feeds are then used as a source of correlations for all the events and attributes without the need for the organization to import them directly into their system. Cyber threat feeds will be collected as Comma Separated Values (CSV), free text or JSON. CSV will allow for the specific cyber attributes to be selected before processing while free-text formats provide unstructured indicators to be added to the threat intelligence system. At this level, advanced filtering capability will be enabled and applied by end-users.

Step 3 perform an analysis using supervised ML methods for the identification and categorization of incidents and events.

Step 4 is where security breaches are identified with the aid of intuitive GUls for end-users to collaborate on events and threat indicators. The GUI helps navigate seamlessly between events and their correlations but also shows alerts and remediation suggestions. In step 4 the technology shows security orchestration and automation where threats can be automatically blocked that either the stakeholders or the community have blocked.

Figure 4 showed how threat data is collected, processed, analysed and shared with other organizations. Threat data is automatically made available to participating organisations via the client GUI that is installed at the organisation's premises. The exchange is made possible by threat sharing protocol STIX which defines what intelligence is shared (Sauerwein, et al., 2017). The platform can thus synchronize threat intelligence and distribute it to different application clients seamlessly. Additionally, the platform will share its data with other threat repositories such as the MISP or the CIRCL. Since the data collected is structured, many existing incident management and SIEM tools can ingest this data directly.

\section{Standards and Legislative Compliance}

Various cybersecurity standards and frameworks address security hardening, incident response etc. For example, the ISO 27001 standard provides specifications that assist organizations in designing, implementing, reviewing, maintaining, and continuously improving an ISO 27001 compliant ISMS (Bawono, et al., 2020). The National Institute for Standards and Technology (NIST) cybersecurity framework provides broad guidelines and 
best practices that ensure a well-built, and continuous improvement of any organization's security posture (Gordon, et al., 2020). Security activities within the NIST framework are categorised according to five basic functions. These are Identify, Protect, Detect, Respond, and Recover (see Table 1). The CTI exchange platform discussed in this paper is designed to support these initiatives and form collaboration between collaborators by sharing loCs, Tactics, Techniques and Procedures (TTP), remediations etc.

Table 1: NIST Framework Functions

\begin{tabular}{|l|l|}
\hline Function & Description \\
\hline Identify & The Identify focuses on identifying critical organization assets and processes. \\
\hline Protect & $\begin{array}{l}\text { The protect function involves specifying, as well as implementing security mechanisms to protect the critical } \\
\text { assets and processes. }\end{array}$ \\
\hline Detect & \begin{tabular}{l} 
The organizations detect function outline activities to identify occurrences of cyber-attacks. \\
\hline Respond
\end{tabular} $\begin{array}{l}\text { The organization's response function focuses on procedures to be followed once a cyber-incident is } \\
\text { detected, this includes but is not limited to roles and responsibilities and limiting the impact of the cyber } \\
\text { incident. }\end{array}$ \\
\hline Recovery & $\begin{array}{l}\text { The organization's recovery function conducts recovery planning processes and procedures to restore } \\
\text { systems and/or assets damaged by cybersecurity events. }\end{array}$ \\
\hline
\end{tabular}

In 2015 the SA government published the National Cybersecurity Policy Framework (NCPF). Its purpose was to establish a safe and secure cyber environment that emphasizes the protection of critical information infrastructure. For this to be achieved a joined force between different stakeholders (civil society, private, and public sectors respectively) is necessary, in understanding as well as addressing risks within cyberspace. (Fonseca, et al., 2021) Through the guidance of NCPF, a national cybersecurity hub, which is SA's National Computer Security Incident Response Team (CSIRT) was established. The CSIRT identifies and counters cyber threats.

SA's Protection of Personal Information (POPIA) is strictly intended to set out conditions on how and when an organization is allowed to process ("including collecting, using, transferring, matching and storing" (Rachel, et al., 2021)) personal information. SA organizations are required to ensure that they comply with the ACT by July 2021. The ACT consist of three role players, that is the Data Subject, Responsible Party, and the Operator. Amongst its compliance requirements, the POPIA specifies that responsible parties should report data breaches, only share information when they are lawfully allowed, and verify if they are lawfully authorized to transfer PII. The technology presented here will not be consuming any PII from the networks it is deployed on. Furthermore, the organization decides what information it will share with other collaborators.

\section{Conclusion}

Cybercriminals are sharing tools and information. Cybercriminals also sell exploits and tools to each other. Ransomware-as-a-service. Organizations in the same industries with common goals should do the same to defend against cyber-attacks, the platform presented here shows that such a system could be developed. Organizations will have a way to share cybersecurity intelligence, while their private data remains private, and in some cases should an organization wish to share their data they can decide what to share and with whom. This intelligence will provide a contextualized security posture about the network environment and specific threats that are relevant to each industry. The collaboration amongst the industry players would yield higher defences against threat actors. Since their TTP's would have been shared and exposed to a wider audience. Our system will have the following capabilities: store, analyse, share, collaborate on cybersecurity indicators, malware analysis, and use the loCs and information to detect and prevent attacks or threats against ICT infrastructures and organisations. While providing stakeholder and expert level reporting and analysis. A CTI exchange platform should reduce data duplication and the effort required for the analysis and classification of potential threats. The solution's main function is to create a CTI repository in SA which will assist collaborators with identifying, protecting, and responding to cyber threats related to their industry. Future work will involve the implementation of how the gathered intelligence can be converted to different formats that can be used in a Security Operations Centre (SOC) together with various threat detection and analysis tools. Improving the GUI to cater for specific industries with similar interests. To continuously find better ways to improve threat detection and intelligence sharing with stakeholders.

\section{References}

Accenture, 2020. Insight into the Cyberthreat Landscape in South Africa, Johannesburg: Accenture. 
Alessandra, d. M. e. S., João José Costa, G., Robson, d. O. A. \& Villalba, L. J. G., 2020. A Methodology to Evaluate Standards and Platforms. Future Internet, Volume 12, p. 108.

Allen, K., 2021. Critical infrastructure attacks: why South Africa should worry. [Online] Available at: https://issafrica.org/iss-today/critical-infrastructure-attacks-why-south-africa-should-worry [Accessed 3007 2021].

Anomali, 2021. Anomali threatstream. [Online] Available at: https://www.anomali.com/products/threatstream [Accessed 25 May 2021]

Bawono, A. et al., 2020. Analysis Corellation of the Implementation Framework COBIT 5, ITIL V3 and ISO 27001 for ISO 10002 Customer Satisfaction. In: s.l.:EasyChair.

Burger-Smidt, A., 2020. Massive data breach in SA - did Experian do enough?. [Online] Available at: https://www.bbrief.co.za/2020/09/09/massive-data-breach-in-sa-did-experian-do-enough/ [Accessed 25 July 2021].

Cascavilla, G., Tamburri, D. A. \& Van Den Heuvel, W.-J., 2021. Cybercrime threat intelligence: A systematic multi-vocal literature review. Computers \& Security, Volume 105, p. 102258.

CDNETWORKS, 2021. THE INDUSTRIES MOST VULNERABLE TO CYBER ATTACKS IN 2021. [Online] Available at: https://www.cdnetworks.com/cloud-security-blog/the-5-industries-most-vulnerable-to-cyber-attacks/ [Accessed 3007 2021].

Cimpanu, C., 2020. Experian South Africa discloses data breach impacting 24 million customers. [Online] Available at: zdnet.com/article/experian-south-africa-discloses-data-breach-impacting-24-million-customers/ [Accessed 25 July 2021].

Cyber Intelligence House, 2020. Cyber Exposure Index. [Online] Available at: https://cyberexposureindex.com/ [Accessed 3107 2021].

CybercrimeSA, 2014-2021. Cybercrime.org.za. [Online] Available at: http://cybercrime.org.za/ [Accessed 2807 2021].

Dandurand, L. \& Serrano, O. S., 2013. Towards Improved Cyber Security Information Sharing, Tallinn: 5th International Conference on Cyber Conflict.

ENISA, 2018. Exploring the opportunities and limitations of current Threat Intelligence Platforms, s.I.: ENISA.

Fonseca, R., Wyk, S. v. \& Jo-Ansie, 2021. Cybersecurity in South Africa: Status, governance, and prospects. In: Routledge Companion to Global Cyber-Security Strategy. s.l.:Routledge.

Frisby, J., 2020. Cybersecurity Exposure Index (CEI) 2020. [Online] Available at: https://passwordmanagers.co/cybersecurity-exposure-index/ [Accessed 31 July 2021].

GADGETS, C., 2021. The FASTEST Way to Consume Threat Intelligence.. [Online] Available at: https://csirtgadgets.com/collective-intelligence-framework [Accessed 4 August 2021].

Gordon, A, L., Loeb, M. \& Zhou, L., 2020. Integrating cost--benefit analysis into the NIST Cybersecurity Framework via the Gordon--Loeb Model. Journal of Cybersecurity.

Kaspersky, 2021. Threat Intelligence Definition. Why Threat Intelligence Is Important for Your Business and How to Evaluate a Threat Intelligence Program. [Online]

Available at: https://www.kaspersky.com/resource-center/definitions/threat-intelligence

[Accessed 31 July 2021].

Lauren, R. \& Irwin, B., 2015. Towards a framework for automated generation, Grahamstown: Rhodes University.

Lord, N., 2020. Digital Guardian. [Online] Available at: https://digitalguardian.com/blog/what-are-indicators-compromise [Accessed 11 August 2021].

MISP Project, 2021. MISP - Open Source Threat Intelligence Platform \& Open Standards For Threat Information Sharing. [Online] Available at: https://www.misp-project.org/ [Accessed 03 August 2021].

Motlhabi, M., Pantsi, P. \& Netshiya, R., 2021. A Machine Learning Deep-dive Analysis into Network logs, Tennessee, US: ICCWS 2021, 6th International Conference on Cyber Warfare and Security.

Mtsweni, J., Mutemwa, M. \& Mkhonto, N., 2016. Development of a cyber-threat intelligence-sharing model from big data sources. Journal of Information Warfare, 15(3), pp. 56-68.

$\mathrm{NCl}$ Agency, 2017. NCl Agency Malware Information Sharing Platform, s.I.: NCl.

OASIS Cyber Threat Intelligence (CTI) TC, 2021. Introduction to TAXII. [Online] Available at: https://oasis-open.github.io/cti-documentation/taxii/intro.html [Accessed 0408 2021].

Rachel, A. et al., 2021. POPIA Code of Conduct for Research. South African Journal of Science, 117(5-6).

Rudman, L. \& Irwin, B., 2016. A sharing platform for Indicators of Compromise. Fancourt, George, Southern Africa Telecommunication Networks and Applications Conference (SATNAC). 
Sari, A., 2018. Context-Aware Intelligent Systems for Fog Computing Environments for Cyber-Threat Intelligence. s.l.:Springer, Cham.

Sauerwein, C., Sillaber, C., Mussmann, A. \& Breu, R., 2017. Threat Intelligence Sharing Platforms: An Exploratory Study of Software Vendors and Research Perspectives. Leimeister, s.n.

Software Testing Help, 2021. Top 11 Best SIEM Tools In 2021 For Real-Time Incident Response And Security. [Online] Available at: https://www.softwaretestinghelp.com/siem-tools/

South African Reserve Bank, 2021. FINANCIAL STABILITY REVIEW. [Online] Available at: https://www.resbank.co.za/en/home/publications/publication-detail-pages/reviews/finstabreview/2021/First-edition-2021-Financial-Stability-Review0 [Accessed 2907 2021].

TechCentral, 2021. Transnet likely hit by Death Kitty ransomware attack. [Online] Available at: https://techcentral.co.za/transnet-likely-hit-by-death-kitty-ransomware-attack-experts/109642/ [Accessed 0508 2021].

The Mitre Corporation, 2016. The Mitre Corporation. [Online] Available at: http://stixproject.github.io/usecases/ [Accessed 1908 2021].

ThreatQuotient, 2021. THREATQ ${ }^{\text {TM }}$ Platform. [Online] Available at: https://www.threatq.com/threatq-platform/ [Accessed 18 June 2021].

Varonis.com, 2021. 134 Cybersecurity Statistics and Trends for 2021. [Online] Available at: https://www.varonis.com/blog/cybersecurity-statistics/ [Accessed 1908 2021].

Wagner, T. D., Mahbub, K., Palomar, E. \& Abdallah, A. E., 2019. Cyber threat intelligence sharing: Survey and research directions. Computers \& Security, Volume 87, p. 101589. 